\title{
Mathematical Transforms in Design: Case Study on Feedback Control of a Customizable Automotive Suspension
}

\author{
Hrishikesh V. Deo ${ }^{1}$, Nam P. Suh ${ }^{1}(1)$ \\ ${ }^{1}$ Dept. of Mechanical Engineering, Massachusetts Institute of Technology, Cambridge, MA 02139
}

\begin{abstract}
Engineering design and analysis is replete with examples of mathematical transforms. This paper discusses the use of mathematical transforms at the operational stage implemented by superimposing the system with a control system to (1) convert a decoupled or coupled system to uncoupled, (2) achieve robustness to noise factors and (3) eliminate imaginary complexity. This paper proves with examples that such controller design and implementation is much easier for an uncoupled or decoupled design as compared to a coupled design. The case study presents a new customizable automotive suspension with independent control of stiffness, damping and ride-height. This system was proposed, designed and built using axiomatic design principles. The mechanical design is decoupled with respect to the functional requirements (FRs) of stiffness and rideheight; moreover ride-height is affected by the load on the vehicle (noise factor). This paper presents the design and implementation of a feedback control system for the customizable suspension to uncouple the system and to make it robust to the noise factor.
\end{abstract}

\section{Keywords:}

Axiomatic Design, Suspension system, Feedback

\section{INTRODUCTION}

Mathematical transforms are frequently employed in design process at different stages; conceptual design, parametric design and even during the operation of the designed system. For instance, the frequently encountered second order differential equation of the form $M \ddot{x}+B \dot{x}+K x=F$ is invariably converted to $\ddot{x}+2 \zeta \omega_{n} \dot{x}+\omega_{n}^{2} x=f$ using the transform $\omega_{n}=\sqrt{K / M}$ and $\zeta=B / 2 \sqrt{K M}$. This is essentially the transformation from the physical DPs $\{B K\}^{T}$ to intermediate DPs $\left\{\zeta \omega_{n}\right\}^{T}$ to aid the designer as they are trained to think in terms of these intermediate DPs. These intermediate DPs are most widely used when the FRs are uncoupled or decoupled with respect to the intermediate DPs, whereas they may be coupled with respect to the physical DPs. For instance, in the example above, the FRs of percent overshoot and peak time are decoupled with respect to the intermediate DPs $\left\{\zeta \omega_{n}\right\}^{T}$, whereas they are coupled with respect to the physical DPs $\{B K\}^{T}$. Another example is the introduction of dimensionless parameters in thermal and fluid sciences [1]. The physical DPs like density, velocity, viscosity and length scales are transformed to intermediate DPs such as the Reynolds number to aid the designer develop insight.

Another common use of mathematical transforms is at the operational stage of the designed system. Open-loop control systems can be used to convert a coupled or decoupled system to an uncoupled system, but such application is restricted due to presence of noise factors and limited knowledge of the system. Feedback control systems can be used to achieve this uncoupling as well as robustness to noise factors. This paper illustrates, with examples, how open-loop or feedback control can be used to implement such a transform and the potential benefits. The case study describes the development of a decoupled customizable automotive suspension and design of feedback control to convert it to an uncoupled system, which is also robust to noise factors. The effectiveness of mathematical transforms implemented at the operational stage is illustrated through this example.

\section{INTRODUCTION TO AXIOMATIC DESIGN [2]}

Axiomatic design is a structured design method created to improve design activities by establishing criteria on which potential designs may be evaluated and by developing tools for implementing these criteria. Axiomatic design discusses the existence of four domains in the design world- customer, functional, physical and process domains. Customer attributes $\{C A s\}$, functional requirements $\{F R s\}$, design parameters $\{D P s\}$, and process variables $\{P V s\}$ are the characteristic vectors of these domains. Design of products involves mapping from the functional domain to the physical domain and design of processes involves mapping from the physical domain to the process domain.

The axiomatic design process is centered on the satisfaction of FRs, which are defined as the minimum set of independent requirements that completely characterize the functional need of the product. Given a minimum set of independent FRs, the designer conceives a physical embodiment or a design containing a set of DPs, which are key physical variables in the physical domain that characterize the design that satisfies the specified FRs. The design and the choice of DPs are guided by the two design axioms.

- Axiom 1: Independence Axiom- Maintain the independence of all functional requirements.

- Axiom 2: Information Axiom- Minimize the information content of the design.

The design matrix (DM) is used to note the effect of DPs on FRs as follows:

$$
\left\{\begin{array}{l}
\mathrm{FR} 1 \\
\mathrm{FR} 2
\end{array}\right\}=\left[\begin{array}{cc}
\mathrm{A}_{11} & \mathrm{O} \\
\mathrm{A}_{21} & \mathrm{~A}_{22}
\end{array}\right]\left\{\begin{array}{l}
\mathrm{DP} 1 \\
\mathrm{DP} 2
\end{array}\right\}
$$

where $A_{11}$ denotes the effect of DP 1 on FR $1, A_{21}$ denotes the effect of DP 1 on FR 2, etc. To satisfy the Independence Axiom, the DM must be must be either 
diagonal or triangular. In an uncoupled design, the DM is diagonal and each of the FRs can be satisfied independently by adjusting one DP. In a decoupled design, the matrix is triangular and the independence of FRs can be guaranteed only if the DPs are determined in a proper sequence. In the case shown, DPs should be set in the order: DP 1 followed by DP 2. A full design matrix leads to a coupled design and the satisfaction of FRs becomes difficult.

The Information Axiom guides the designer to maximize the probability of satisfaction of the FRs. It becomes increasingly difficult to satisfy FRs when FRs are coupled by the chosen DPs. This is because the allowable tolerance for DPs decreases with the increase in the number of FRs and the number of off-diagonal elements in the design matrix.

\section{IMPLEMENTATION OF MATHEMATICAL TRANSFORMS}

\subsection{Open loop control (Inversion of DM)}

Equation 2 represents a linear time-invariant and physically decoupled design, not affected by any noise factor. DP1 and DP2 are the operational DPs that the user varies during system operation to set the FRs.

$$
\left\{\begin{array}{l}
\mathrm{FR} 1 \\
\mathrm{FR} 2
\end{array}\right\}=\left[\begin{array}{ll}
\mathrm{A} & \mathrm{O} \\
\mathrm{B} & \mathrm{C}
\end{array}\right]\left\{\begin{array}{l}
\mathrm{DP} 1 \\
\mathrm{DP} 2
\end{array}\right\}
$$

This system has the following disadvantages. First, the DPs have to be set in a particular order to maintain the independence of the FRs; also a change in DP1 necessitates a change in DP2 to compensate for change in FR2 due to DP1. Second, without the knowledge of the $\mathrm{DM}$, there is an imaginary complexity [3] involved in finding the correct order of setting the DPs. Imaginary complexity becomes increasingly important as the number of FRs ( $n$ ) increases, as the probability of finding the correct sequence of setting DPs for a decoupled design without knowledge of the design interactions is $1 /(n !)$.

If the physically decoupled system shown by equation 2 is superimposed with a control system having a $\mathrm{DM}_{\text {controller }}$ (equation 3 ), that is the inverse of the original DM in equation $2(X=1 / A, Z=1 / C$ and $Y=-B X / C)$, then we get $a$ resultant uncoupled $D M$ as shown in equation 4 .

$$
\begin{aligned}
& \left\{\begin{array}{l}
\mathrm{DP} 1 \\
\mathrm{DP} 2
\end{array}\right\}=\left[\begin{array}{ll}
\mathrm{X} & \mathrm{O} \\
\mathrm{Y} & \mathrm{Z}
\end{array}\right]\left\{\begin{array}{l}
\mathrm{DP}_{\mathrm{user}} 1 \\
\mathrm{DP}_{\mathrm{user}} 2
\end{array}\right\} \\
& \left\{\begin{array}{l}
\mathrm{FR} 1 \\
\mathrm{FR} 2
\end{array}\right\}=\left[\begin{array}{ll}
1 & \mathrm{O} \\
\mathrm{O} & 1
\end{array}\right]\left\{\begin{array}{l}
\mathrm{DP}_{\mathrm{user}} 1 \\
\mathrm{DP}_{\mathrm{user}} 2
\end{array}\right\}
\end{aligned}
$$

This implementation of mathematical transform, by superimposing the design with an open-loop control system, makes the decoupled system appear uncoupled to the user during operation (equation 4). It also eliminates the imaginary complexity as any sequence of setting the DPs is acceptable. This mathematical transform of inversion could be implemented by a control system, hardware or even by the user while the system is in use. For instance, a hot water faucet is a coupled design with the hot water knob position $(\mathrm{H})$ and cold water knob position (C) as the DPs as shown by equation 5 . The user can make this system behave as uncoupled during operation as shown in equation 6 . By moving the hot water knob and the cold water knob by the same amounts $(\mathrm{H}+\mathrm{C})$, the user can change only flow rate and not temperature; and by moving the hot water knob and cold water knob by opposite amounts $(\mathrm{H}-\mathrm{C})$, the user can change only temperature and not flow rate. The same transform could be achieved through hardware or a control system.

$$
\begin{aligned}
& \left\{\begin{array}{c}
\text { FR1: Flow Rate } \\
\text { FR2: Temperature }
\end{array}\right\}=\left[\begin{array}{ll}
\mathrm{X} & \mathrm{X} \\
\mathrm{X} & \mathrm{X}
\end{array}\right]\left\{\begin{array}{l}
\mathrm{DP} 1: \mathrm{H} \\
\mathrm{DP} 2: \mathrm{C}
\end{array}\right\} \\
& \left\{\begin{array}{c}
\text { FR1: Flow Rate } \\
\text { FR2: Temperature }
\end{array}\right\}=\left[\begin{array}{ll}
\mathrm{X} & \mathrm{O} \\
\mathrm{O} & \mathrm{X}
\end{array}\right]\left\{\begin{array}{l}
\mathrm{DP} \mathrm{user}_{\mathrm{DP}} 1: \mathrm{H}+\mathrm{C} \\
\mathrm{DP}_{\text {user }} 2: \mathrm{H}-\mathrm{C}
\end{array}\right\}
\end{aligned}
$$

In theory, the DM for a coupled system can also be inverted and used to implement this mathematical transform for a coupled system, assuming that the system is modeled accurately and noise factors are not present. Inversion of DMs for coupled design is more involved than decoupled systems, but nothing can be said about performance comparisons in the absence of noise factors.

\subsection{Closed loop control}

Note that application of open loop control to invert the $\mathrm{DM}$, discussed above, is restricted only to linear systems not affected by noise factors. But in real-life design situations, noise factors always affect FRs and a common alternative is the use of feedback control to compensate for the noise factors. This feedback could be either manual or automatic. For manual control, it is easy to see that uncoupled designs are far easier to control than coupled designs, more so as the number of FRs or the degree of coupling increases. For automatic feedback control, an uncoupled system can be considered as an integration of SISO systems and implementation of such a feedback system is straightforward with classical control schemes. Decoupled systems can also be looked at as SISO systems, if the FRs (or DPs) are set in a particular order. Coupled systems present themselves as MIMO systems, require modern control techniques and are significantly more difficult to control. Moreover, feedback control of coupled systems requires state feedback and accurate modeling of the system, whereas only output feedback and coarse modeling suffices for uncoupled or decoupled systems. This illustrates the importance of the Independence Axiom, which guides the designer to come up with uncoupled or decoupled systems, which are easily amenable to either manual or automatic "measure and compensate". Hence uncoupled or decoupled designs are far superior in operation to coupled designs.

\section{CASE STUDY}

\subsection{Motivation for customizable suspension}

Design of automotive suspension systems typically involves a trade-off between the conflicting requirements of comfort and handling. For instance, cars need a soft suspension for good comfort, whereas a stiff suspension leads to better handling and attitude control. Cars need high ground clearance on rough terrain, whereas a low center of gravity height is desired for swift cornering and dynamic stability at high speeds. It is advantageous to have low damping for low force transmission to vehicle frame, whereas high damping is desired for fast decay of oscillations. To avoid these trade-offs, we have proposed, designed and built a customizable suspension system with independent control of ride-height, stiffness and damping. The user can set the stiffness, damping and ride-height according to his choice to get desired performance from the car. Damping control, typically achieved through orifice control, is an established technology in existing vehicles [4,5]. Several road vehicles with pneumatic springs are capable of achieving variable ride-height [6]. Advantages of variable stiffness have been illustrated in literature [7], but no system with independent control of stiffness and ride-height has been proposed. Designs, proposals, benefits and 
disadvantages of advanced ground vehicle suspension systems have been reported in the form of a classified bibliography [8]. The design and working of the proposed adaptive suspension system is explained in the next subsection.

\subsection{Proposed design of customizable suspension}

Figure 1 shows the kinematic representation of a SLA (short long arm) suspension, which is the most widely used architecture for independent front-wheel suspension. Variable stiffness and ride-height can be achieved by making the lower and upper spring pivots movable. Figure 2 shows one possible mechanism for achieving this. The lower spring pivot is driven by a linear stage, consisting of a stepper motor, a lead screw and a linear bearing. Motion of the lower spring pivot changes the effective stiffness $\mathrm{K}_{\mathrm{w}}$ by changing the relation between the wheel travel and spring deflection. The effective stiffness seen at the wheel $K_{w}$ is related to the spring stiffness $K_{s}$ and DP1: $x$ as given by equation 7 . Ride-height can be changed by moving the upper pivot by a motor driven cam. Movement of the upper pivot (lift of the cam) is used as DP2: $U$ as shown in the design matrix in equation 8. FR3: Control damping is achieved by DP3: Orifice control. The damper (not shown in the figure for clarity) is connected in parallel with the spring. Since FR3 is independent and DP3 doesn't affect any other FR, we will neglect this FR/DP pair in the subsequent analysis for simplicity.

$$
K_{w}=K_{s}\left(\frac{x}{L}\right)^{2}
$$

$$
\left\{\begin{array}{c}
\text { FR1: Stiffness } \\
\text { FR2: Ride-height } \\
\text { FR3: Damping }
\end{array}\right\}=\left[\begin{array}{ccc}
\mathrm{X} & \mathrm{O} & \mathrm{O} \\
\mathrm{X} & \mathrm{X} & \mathrm{O} \\
\mathrm{O} & \mathrm{O} & \mathrm{X}
\end{array}\right]\left\{\begin{array}{c}
\mathrm{DP} 1 \text { : Pivot position } \mathrm{x} \\
\mathrm{DP} 2 \text { : Cam position } \mathrm{U} \\
\mathrm{DP} 3: \text { Orifice control }
\end{array}\right\}
$$

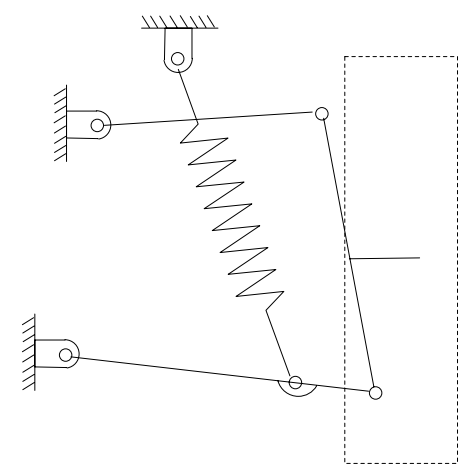

Figure 1: Kinematic representation of existing independent SLA suspension

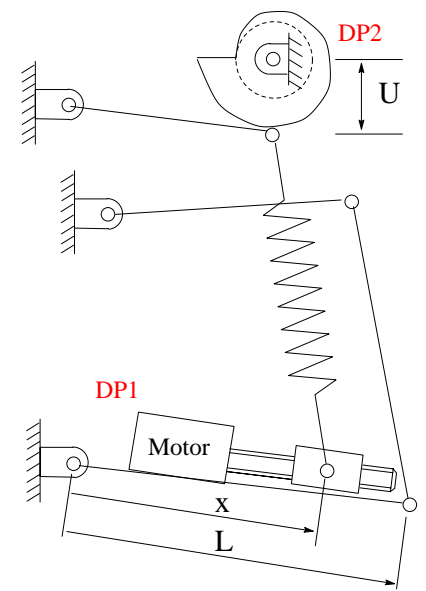

Figure 2 : Proposed modifications to achieve independent control of stiffness and ride-height
FR2: Ride-height depends not only on the cam position $U$ (DP2), but also on stiffness (hence on DP1), and load on the vehicle (noise factor $D P_{n f}$ ). Note that $D P_{n f}$ is not a $D P$ that the user can set, but is introduced in the design equation to indicate effect of the noise factor $\left(D P_{n f}\right)$ on FRs [9]. To study the effect of the DPs on FR2: Rideheight, the system is modeled as a single degree of freedom (DOF) quarter car model as shown in Figure 3. The actuator (motor driven cam) is modeled as a low frequency displacement provider. The actuator provides displacement U (DP2) in series with the spring. The block diagram in Figure 4 shows the transfer functions relating the output $X_{s}$ to the inputs to the system: road noise $X_{r}$, load on vehicle $F$ and cam displacement U. Equation 9 shows that this is a decoupled system (due to offdiagonal term C) and FR2: Ride-height is affected by the $\mathrm{DP}_{\mathrm{nf}}$ : Load on the vehicle (term D).

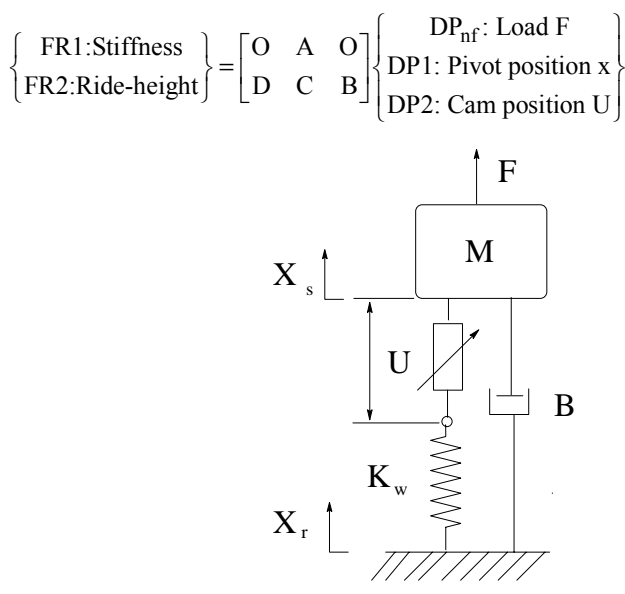

Figure 3 : Representation of the system as a quarter-car 1-DOF system

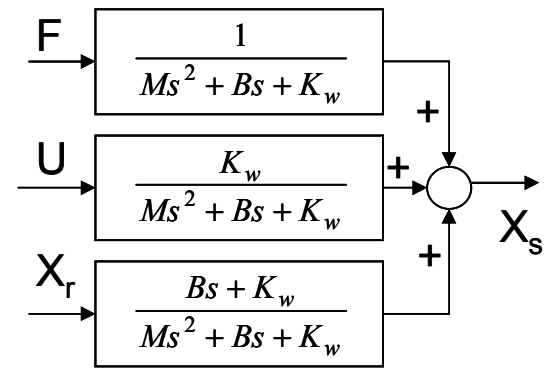

Figure 4 : Block diagram representation of the proposed system

\subsection{Implementation of feedback}

FR1: Stiffness is not affected by any noise factor; hence we have used open loop control of stiffness. Equation 7 is used to calculate position of the lower pivot (DP1: $x$ ) from the desired value of FR1: Stiffness $K_{w}$.

The off-diagonal terms $C$ and $D$, in the DM of equation 9 , indicate the dependence of ride-height on stiffness change and load change respectively. Any change in DP1: stiffness setting or $\mathrm{DP}_{\mathrm{nf}}$ : load necessitates a change in DP2 by the user to maintain ride-height constant. To achieve insensitivity to stiffness change and load change, a feedback control system for ride-height was designed as shown in Figure 5. Since the system is decoupled and we set the DPs in appropriate order, we can treat ride-height control as a SISO system. This enables the use of classical control techniques treating DP2: $U$ as input to the plant and $K_{w}$ and $F$ as noise factors. The actual ride-height $\left(X_{s}-X_{r}\right)_{\text {actual }}$ is measured and compared with the desired ride-height $\left(X_{s}-X_{r}\right)_{\text {desired. }}$ An encoder connected to the suspension control arm gives a 
measurement for $\left(X_{s}-X_{r}\right)_{\text {actual }}$. The controller determines the desired value for DP2: $\cup_{\text {des }}$ according to a control law based on the difference between the actual and desired ride-height values. The controller in the customizable suspension prototype built at MIT is a $\mathrm{PI}$ controller in series with a low pass filter. PI controller is used for zero steady state error. The low-pass filter is to filter out the high frequency component of the actual ride-height change: $\left(X_{s}-X_{r}\right)_{\text {actual }}$ caused due to road-noise.

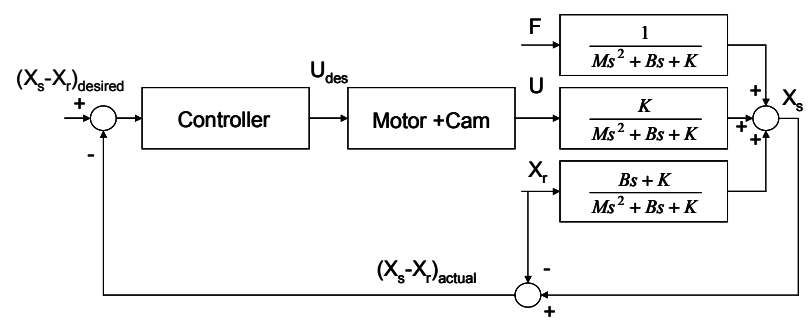

Figure 5 : Ride-height feedback control

Representation of the resultant system in equation 10 shows that after introducing the feedback control, the decoupled system is converted to uncoupled. Note the difference between DP2: Cam position $U$ in equation 9 and $\mathrm{DP}_{\text {user }}$ 2: Ride-height command $\left(\mathrm{X}_{\mathrm{s}}-\mathrm{X}_{\mathrm{r}}\right)_{\text {desired }}$ in equation 10 . Also equation 10 shows that ride-height is independent of the load on the vehicle and hence this transform has also achieved robustness to a noise factor. Also the imaginary complexity is eliminated as the system appears uncoupled to the user during operation. With minimal hardware change, the physically decoupled system (equation 9) has been converted to a system (equation 10) that the user sees as uncoupled during the operation of the system!

$$
\left\{\begin{array}{c}
\text { FR1:Stiffness } \\
\text { FR2:Ride-height }
\end{array}\right\}=\left[\begin{array}{ccc}
\mathrm{O} & \mathrm{A} & \mathrm{O} \\
\mathrm{O} & \mathrm{O} & 1
\end{array}\right]\left\{\begin{array}{c}
\mathrm{DP}_{\mathrm{nf}}: \text { Load F } \\
\mathrm{DP}_{\text {user }} 1: \text { Pivot position x } \\
\mathrm{DP}_{\text {user }} 2: \mathrm{RH}\left(\mathrm{X}_{\mathrm{s}}-\mathrm{X}_{\mathrm{r}}\right)_{\text {desired }}
\end{array}\right\}
$$

\section{CONCLUSIONS}

A novel customizable suspension system with independent control of stiffness, ride-height and damping has been proposed. This system when used in open loop is decoupled with respect to the FRs of stiffness and rideheight; moreover the ride-height is affected by the load on the vehicle (noise factor). A feedback control system for ride-height control is designed and implemented for this decoupled system. This implementation of mathematical transforms makes the system uncoupled with respect to stiffness and ride-height, and also achieves robustness to noise factor (load on the vehicle).

This illustrates an interesting use of mathematical transforms during operation of a design to uncouple a physically decoupled system, to achieve robustness to noise factors and to eliminate the imaginary complexity from the system. It is further shown that such mathematical transforms are very simple to design and implement for uncoupled or decoupled systems as opposed to coupled systems, thereby illustrating the importance of the Independence Axiom.

Superposition by an open-loop control system during operation and introduction of intermediate DPs during conceptual and parametric design stage are also presented as decoupling or uncoupling strategies.

\section{SUMMARY}

The use of mathematical transforms in design is illustrated to achieve uncoupling or decoupling and robustness. Introduction of intermediate DPs during conceptual and parametric design, and superposition by open loop control or feedback control during operation are cited as means to achieve these mathematical transforms. The case study presents the axiomatic design of a new customizable automotive suspension with independent control of stiffness, damping and ride-height.

\section{ACKNOWLEDGMENTS}

This research was carried out with funding from the FordMIT alliance. We extend our sincere thanks to Dr. Tim Davis of Ford Motor Company and Dr. Jason Melvin of Axiomatic Design Research Group, MIT for valuable suggestions and contribution to the project.

\section{NOMENCLATURE}

FR: Functional Requirement

DP: Design Parameter

DM: Design Matrix

SISO: Single Input Single Output

MIMO Multi Input Multi Output

\section{REFERENCES}

[1] Taylor, E.S., 1974, Dimensional Analysis for Engineers, Clarendon Press, Oxford.

[2] Suh, N.P., 2001, Axiomatic Design: Advances and Applications, CIRP Design Book Series, Oxford University Press, Oxford, NY.

[3] Suh, N.P., 2004, Complexity: Theory and Application, Oxford University Press, Oxford, NY.

[4] Karnopp, D.C., 1983, Active damping in road vehicle suspension systems, Vehicle System Dynamics, 12, 291-316.

[5] Crosby, M.J., Karnopp, D.C., 1973, The active damper- a new concept for shock and vibration control.

[6] Cho, D., Hedrick, J.K., 1985, Pneumatic actuators for vehicle active suspension applications, Trans. A.S.M.E. Journal of Dynamic Systems, Measurement and Control, 107, 67-72.

[7] Karnopp D., Margolis D., 1984, Adaptive Suspension Concepts for Road Vehicles, Vehicle System Dynamics, 13, 145-160.

[8] Elbeheiry, E.M., et.al., 1995, Advanced Ground Vehicle Suspension Systems - A Classified Bibliography, Vehicle System Dynamics, 24, 231258.

[9] Melvin, J.W., Deo, H.V., 2002, Axiomatically Designed Robustness, American Supplier Institute 19th Annual Taguchi Methods Symposium, San Diego, CA. 